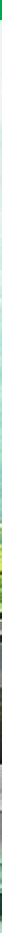

Interview mit Prof. Dr. med. Jürg Kesselring, Präsident des Swiss Brain Council

\title{
«Es ist zentral, eine verständliche Sprache zu sprechen»
}

\author{
Mit dem Swiss Brain Council (SBC) besteht seit kurzem eine gesamtschweizerische \\ Organisation, in der Interessengruppen aus den Bereichen Klinische Neurologie \\ und Neurowissenschaften, aber auch Patientenorganisationen vereint sind. Präsi- \\ dent Jürg Kesselring erläutert im Gespräch die Idee, die hinter dem SBC steht.
}

Interview: Bruno Kesseli

\begin{abstract}
Im Bereich Neurologie/Neurowissenschaften gibt es schon eine ganze Reihe von Organisationen und Fachgremien. Braucht es da noch einen «Swiss Brain Council»?

Jürg Kesselring: Der Anstoss zur Gründung des Swiss Brain Council kam von dessen europäischem Pendant, dem European Brain Council. Die Idee war, in der Schweiz etwas Ähnliches ins Leben zu rufen,
\end{abstract}

«Eine umfassende Allianz, wie sie nun

mit dem Swiss Brain Council gebildet wurde, gab es in der Schweiz bisher nicht.»

nämlich eine Organisation, in der sowohl die klinisch tätigen Neurologen als auch die Neurowissenschaftler und die Patientenorganisationen vertreten sein sollten. Zwar gab es in der Schweiz verschiedene Dachorganisationen wie die Swiss Federation of
Clinical Neurosocieties SFCNS für den klinischen Bereich oder die Swiss Society of Neuroscience, in der die Neurowissenschaftler organisiert sind. Und es gibt Patientenorganisationen wie die Multiple Sklerose Gesellschaft, Parkinson- und AlzheimerVereinigung usw. Eine umfassende Allianz, wie sie nun mit dem Swiss Brain Council gebildet wurde, gab es in der Schweiz bisher nicht.

Was sind die grundsätzlichen Ziele des Swiss Brain Council?

Einerseits soll der SBC eine Art Lobbyorganisation sein und als Ansprechpartner für die Politiker und die Bevölkerung zur Verfügung stehen. Dabei ist es wichtig, dass es gelingt, sich in einer allgemein verständlichen Art auszudrücken und so die Neurowissenschaften und die Krankheiten, die das Nervensystem betreffen, bekannter zu machen. Ein weiteres Anliegen, das ich persönlich sehr hoch gewichte, ist der Dialog zwischen Grundlagenwissenschaftlern und Klinikern. 


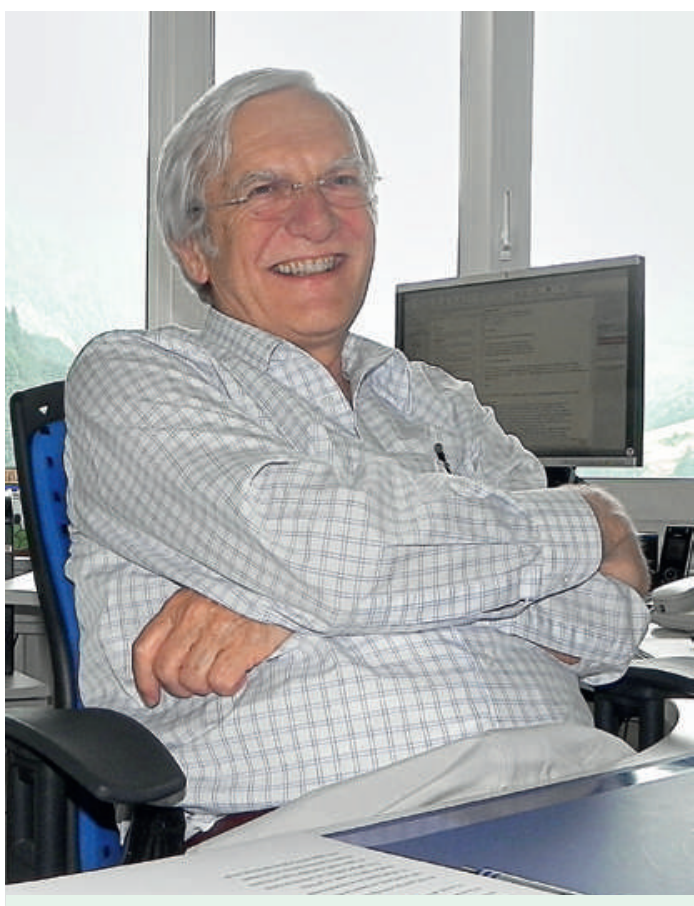

«Voneinander lernen»: Jürg Kesselring ist der Dialog zwischen Klinikern und Grundlagenwissenschaftlern ein zentrales Anliegen.

\section{Warum ist Ihnen dieser Dialog so wichtig?}

Weil wir voneinander lernen, uns gegenseitig in der jeweiligen Tätigkeit anregen können! Ich habe dies eindrücklich erlebt im Rahmen des Nationalen Forschungsprogramms «Krankheiten des Nervensystems», des NFP 38, das von 1993-2001 lief. Eine Auflage war damals, dass Grundlagenwissenschaften und Klinik zusammenkommen und dass Universitäts- und andere Kliniken zusammenarbeiten müssten. Das ist in diesem Rahmen gut gelungen, und das

\section{Swiss Brain Council}

Der Swiss Brain Council (SBC) entstand auf Initiative des European Brain Council. Offiziell gegründet wurde der SBC am 6. Juni 2013 anlässlich des «2nd Congress of the Swiss Federation of Clinical Neuro-Societies» (SFCNS) in Montreux. Der Swiss Brain Council versteht sich als Interessenvertretung zum Thema Gehirn. Er trägt der Wichtigkeit von Gehirnerkrankungen Rechnung und fördert die Erforschung derer Krankheitsbilder. Der SBC ist offen für alle Interessierten aus den Bereichen Medizin, Industrie und Öffentlichkeit sowie für alle von Hirnerkrankungen Betroffenen. Damit ist eine Plattform geschaffen, um aktive Lobbyarbeit in Richtung Regierung und Politik zu betreiben, sowie Journalisten, Politikern und Vertretern der Wirtschaft Erkenntnisse der Gehirnforschung und Folgen der Gehirnerkrankungen näherzubringen.

\section{Jürg Kesselring}

Prof. Dr. med. Jürg Kesselring ist Chefarzt für Neurologie und Rehabilitation am Rehabilitationszentrum in Valens. Er lehrt seit 1996 als Titularprofessor für Klinische Neurologie und Neurorehabilitation an der Universität Bern sowie seit $1998 \mathrm{im}$ Bereich Neurowissenschaften an der Universität Zürich und an der ETH Zürich. 2005-2012 war Jürg Kesselring Präsident der Schweizerischen Multiple Sklerose Gesellschaft. Im Dezember 2010 wurde er zum Mitglied des Internationalen Komitees vom Roten Kreuz (IKRK) kooptiert, für das er zwischen 1978 und 1982 im Rahmen mehrerer Missionen als Arzt tätig war. Von 2008-2011 war Jürg Kesselring zudem Präsident der Sankt-gallischen Kulturstiftung. Neben zahlreichen Fachpublikationen hat er als Autor unter dem Titel «Leise Laute» und «Mit anderen Worten ...» im Basler Verlag Johannes Petri Gedichtbände veröffentlicht.

Programm war nicht zuletzt deshalb meines Erachtens sehr erfolgreich.

Sind denn solche Kontakte nicht ohnehin institutionalisiert?

Aus meiner Sicht besteht in diesem Bereich Verbesserungsbedarf, auch wenn es punktuell immer wieder interessante Initiativen gibt. Zum Beispiel führen wir selbst in Valens seit 15 Jahren die Retraite des in Zürich beheimateten Zentrums für Neurowissenschaften durch. Die Wissenschaftler dieses Zentrums kommen einmal im Jahr für drei Tage hierher, und sie schätzen es besonders, dass ihnen auch Patienten vorgestellt werden. Wenn der Dialog nicht aktiv gepflegt wird, besteht die Gefahr, dass man in Klischees verhaftet bleibt. Aus der Perspektive des Wissenschaftler kann dies dann zugespitzt etwa so tönen: «Ja, die Klinik ist halt eher etwas für die Dummen, die kümmern sich um Blasenprobleme, Schuhe und Ähnliches.» Als Kliniker würde man dagegenhalten: «Ach, diese Wissenschaftler mit ihren Mäusen und Fadenwürmern ...». Und das ist eben schade, weil es in beiden Bereichen viel Neues und Spannendes gibt.

Sie scheinen sich gerne als Brückenbauer zu betätigen. War das der Grund, dass Sie zum Präsidenten des Swiss Brain Council gewählt wurden?

Vermittlerfunktionen sind mir immer ein wichtiges Anliegen. Ich glaube, dies liegt nicht daran, dass ich mich nicht festlegen könnte, sondern dass ich die Menschen mit ihren Verschiedenheiten gern habe. Mitgespielt hat vielleicht auch, dass ich geographisch in der Peripherie tätig bin und dadurch weniger als Interessenvertreter eines Zentrums wahrgenommen werde. 


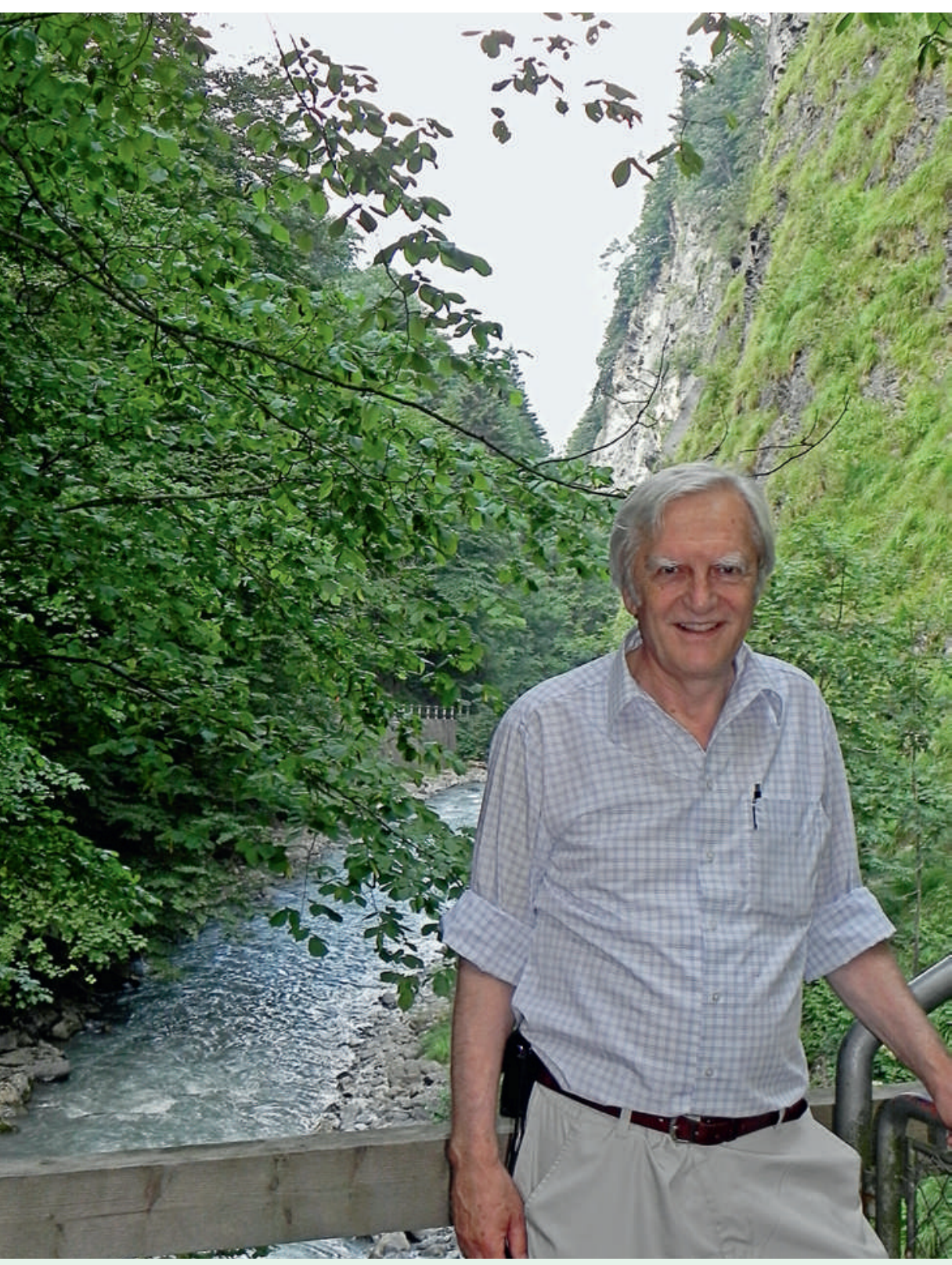

Geschichtsträchtiger Arbeitsweg: Nicht selten durchwandert Jürg Kesselring nach getaner Arbeit die Taminaschlucht.
Schweiz 2014 ein «Year of the Brain» organisieren, wie es auch für Europa geplant ist. Für Studenten wollen wir beispielsweise Fortbildungsangebote im Internet ausbauen, bei denen klinische Fallvorstellungen mit der Vermittlung von theoretischem Hintergrundwissen kombiniert werden.

Laienorganisationen, Kliniker und Grundlagenwissenschaftler im selben Boot: Sind da nicht Interessenkonflikte vorprogrammiert?

Gewisse Ängste sind diesbezüglich sicher vorhanden, zum Beispiel, dass bei der Bearbeitung des «Sponsoringmarktes» Konkurrenzen entstehen können. Auch da wird es unsere Aufgabe sein, zu vermitteln. Ganz wichtig ist auch, eine gemeinsame Sprache zu finden - und diese ist nicht einfach gegeben, sondern muss im Dialog entwickelt werden. Wenn

\section{«Wenn ich verstanden werden will, darf ich nicht einfach vorausset- zen, dass mein Gegenüber meine Sprache schon kann.»}

ich verstanden werden will, darf ich nicht einfach voraussetzen, dass mein Gegenüber meine Sprache schon kann. Ich muss eine Sprache finden, die dem Gesprächspartner angemessen ist. Diese Sprachpflege ist etwas ganz Wichtiges, keinesfalls nur eine Koketterie, die zum Beispiel darin bestehen kann, in der Freizeit ein wenig Gedichte zu schreiben.

Am 26. August fand in Bern ein erstes Arbeitstreffen mit Vertretern der im Swiss Brain Council zusammengeschlossenen Gruppierungen statt. Was wurde dort erreicht?

Neben der Zusammenkunft von Vertreterinnen und Vertretern der erwähnten Gruppierungen war auch die Schilderung des «Human Brain Projects», das bereits im Vorfeld wegen seiner Dimensionen und ambitiösen Ziele viel mediale Aufmerksamkeit erfahren hatte, von Wichtigkeit, vor allem im Hinblick auf eventuell mögliche Synergien. Diese sehe ich aber vor allem auch mit dem «Swiss Neuroscience Web», in dem neurowissenschaftliche Ausbildungsprogramme koordiniert werden. Am Horizont leuchtet schon der erste gemeinsame Kongress der SFCNS und der SSN in Basel im Herbst 2016. 